\title{
Distribution and reproductive effects of Wolbachia in stalk-eyed flies (Diptera: Diopsidae)
}

\author{
AHMAD R. HARIRI $\uparrow \S$, J OHN H. WERREN $\ddagger$ \& GERALD S. WILKINSON*† \\ $\dagger$ Department of Biology, University of Maryland, College Park, MD 20742, USA and †Department of Biology, \\ University of Rochester, Rochester, NY 14627, USA
}

\begin{abstract}
Wolbachia are cytoplasmically inherited bacteria capable of altering the reproductive biology of their hosts in a manner which increases their spread within a population. These microbes can cause cytoplasmic incompatibility, parthenogenesis and feminization of genetic males. Because Wolbachia have been associated with female-biased sex ratio distortion, we used a PCR assay to examine 17 species of stalk-eyed flies (Diptera: Diopsidae), two of which exhibit femalebiased sex ratios, for the presence of these microbes. Type A Wolbachia was detected in four diopsid species, three from the genus Sphyracephala, none of which exhibit biased progeny sex ratios. The reproductive effects of the microbe were examined in one of those species, $S$. beccarii, by conducting reciprocal crosses between infected and uninfected strains. In this species, Wolbachia do not cause detectable cytoplasmic incompatibility or reduce host fecundity. In contrast, our results are consistent with an association between the microbes and enhanced male fertility. Possible explanations for the pattern of distribution and effects on male fertility include a predisposition for acquiring Type A Wolbachia by these flies and accommodation by the host genome to bacterial presence.
\end{abstract}

Keywords: cytoplasmic bacteria, Diopsidae, Sphyracephala, stalk-eyed flies.

\section{Introduction}

Wolbachia are a monophyletic group of proteobacteria with two major divisions, Type $\mathrm{A}$ and $\mathrm{B}$, which have been associated with a wide range of reproductive changes in arthropods (Werren et al., 1995a). They are primarily found within the cells of the gonadal tissues of infected individuals (O'Neill, 1995) and are typically inherited by vertical transmission through the maternal cytoplasm (Hoffmann \& Turelli, 1988). Horizontal transmission, especially of Type A Wolbachia, is believed to play an important role in spreading the infection between species and maintaining phylogenetically similar strains in diverse Orders of arthropods (Werren et al., 1995b). Wolbachia have not been found extracellularly and their existence and proliferation appear to be intimately linked with that of their arthropod hosts (O’Neill, 1995).

Wolbachia typically alter the reproductive biology of their hosts in a manner which ultimately

*Correspondence. E-mail: wilkinson@zool.umd.edu §Present address: Brain Research Institute, UCLA, Los Angeles, CA 90095, USA. promotes the spread of the microorganisms. The effects of Wolbachia infection include unidirectional and bidirectional cytoplasmic incompatibility (CI), parthenogenesis, and feminization of genetic males (see Werren, 1997 for review). Wolbachia can have either positive effects on host fecundity (Girin \& Bouletreau, 1995; Stolk \& Stouthamer, 1996; Poinsot \& Mercot, 1997) and fertility (Wade \& Chang, 1995) or negative effects on host fecundity (Hoffmann et al., 1990). The positive or negative consequences of infection may be related to differences in the mode of microbe transmission and maintenance in different groups of organisms. Models of symbiont transmission predict that negative effects on host reproduction will be prevalent in systems where symbiont populations are maintained by horizontal transmission, and positive effects will be more common when vertical transmission dominates (Frank, 1996).

Recently, female-biased sex ratio distortion has been reported in two species of stalk-eyed flies (Diptera: Diopsidae), Cyrtodiopsis dalmanni and C. whitei (Burkhardt \& De La Motte, 1983), and ascribed to X chromosome meiotic drive (Presgraves et al., 1997). 
Because Wolbachia have been associated with female-biased sex ratio distortion, either through parthenogenesis in haplodiploids (Stouthamer et al., 1993) or feminization in diploids (Rousset et al., 1992), we decided to determine whether or not these microbes are present in $C$. dalmanni and $C$. whitei. In order to determine the potential distribution of Wolbachia infection in stalk-eyed flies an additional 15 diopsid species from six genera were also examined. We also report on the reproductive effects of infection in one of those species, Sphyracephala beccarii, which harbours Type A Wolbachia.

\section{Materials and methods}

\section{Collection}

We collected flies from six of the 11 diopsid genera (Feijen, 1989): Teleopsis, Cyrtodiopsis, Diasemopsis, Diopsis, Sphyracephala and Eurydiopsis. These six genera contain over $96 \%$ of the described species and occupy the entire geographical range of diopsids (Steyskal, 1972). All Teleopsis species (breviscopium, rubicunda, quadriguttata) and Cyrtodiopsis species (dalmanni, whitei, quinqueguttata) were collected in January 1989 in peninsular Malaysia. All Diasemopsis (aethiopica, dubia, munroi, silvatica) and Diopsis (apicalis and fumipennis), as well as Sphyracephala munroi, were collected in December 1994 in the Natal Province, South Africa. Sphyracephala brevicornis were collected in October 1994 in Maryland and $S$. detrahens and Eurydiopsis subnotata in January 1996 in peninsular Malaysia.

Sphyracephala beccarii were collected on 22 November 1993 along a stream near Sudwala Caves, $40 \mathrm{~km}$ west of Nelspruit, Transvaal Province, South Africa. Approximately 20 flies were used to establish a laboratory population and were subsequently bred in the laboratory for 10 months prior to the start of this study. We estimate that the flies used in the first antibiotic treatment experiment (see below) were at least three generations removed from those collected in the field.

\section{Screening for Wolbachia}

The presence of Wolbachia was determined for each species by extracting DNA from pooled ovaries of two to four fecund females and then using the polymerase chain reaction (PCR) to amplify a Wolbachia-specific bacterial cell-cycle gene, fts $Z$, sequence (Werren \& Jaenike, 1995). All samples were screened with universal $28 \mathrm{~S}$ primers as a positive control for amplification ability (see Werren et al., 1995a, for details). Bacterial ftsZ DNA amplification was confirmed by measuring product length from an agarose gel. All solutions were filter-sterilized $(0.22 \mu \mathrm{m}$ pore diameter $)$ to reduce potential bacterial contamination. Control DNA samples were prepared from pupae of known infected and uninfected strains of Nasonia vitripennis and compared with amplified products from each stalk-eyed fly species to determine the presence of Wolbachia. Additional amplifications were performed using Type A and Type B specific primers for fts $\mathrm{Z}$ (Werren et al., 1995a).

\section{Antibiotic curing}

Flies were maintained in modified mouse cages with a $12 \mathrm{~L}: 12 \mathrm{D}$ photoperiodic cycle and $30 \mathrm{~min}$ simulated dawn and dusk periods (Lorch et al., 1993). Flies were allowed to feed ad libitum on a standard food medium consisting of puréed maize treated with a commercial mould inhibitor (Wilkinson, 1993). For breeding purposes, $\approx 35-40 \mathrm{~mL}$ of medium was presented in small plastic cups and changed biweekly. Newly eclosed flies were isolated by sex and maintained as virgins until they were reproductively mature and then utilized in reciprocal crosses.

In order to determine the optimum concentration of antibiotic, which would maximize elimination of the infection and minimize the mortality caused by the toxicity of the antibiotic, flies were bred on media treated with $0.5 \mathrm{mg} \mathrm{mL}^{-1}, 1.0 \mathrm{mg} \mathrm{mL}^{-1}$, or $2.0 \mathrm{mg} \mathrm{mL}^{-1}$ concentrations of aqueous tetracycline, prepared by dissolving tetracycline hydrochloride (Sigma T-3383) in water over low heat. Fecund females (3 weeks post eclosion) from these treatment classes were then tested for the presence of infection using the PCR assay described above. These analyses, in addition to the number of pupae produced by five pairs of flies from each of the three tetracycline concentrations, were used to identify the optimum tetracycline concentration for curing.

These results indicated that the optimum concentration of tetracycline was $1.0 \mathrm{mg} \mathrm{mL}$. At this concentration, the infection was eliminated and the average daily pupal production was $8.0 \pm 1.9$ (SE) per pair. At $0.5 \mathrm{mg} \mathrm{mL} \mathrm{m}^{-1}$, average daily pupal production was higher $(10.0 \pm 1.5)$, but the infection was not eliminated. At $2.0 \mathrm{mg} \mathrm{mL} \mathrm{m}^{-1}$, the infection was eliminated, but average daily pupal production was much lower $(2.0 \pm 1.1)$.

Bacteria were eliminated by allowing 20 females and 20 males to mate and oviposit ad libitum for $48 \mathrm{~h}$ on $1.0 \mathrm{mg} \mathrm{mL}^{-1}$ tetracycline-treated medium. Twenty pairs of progeny were then bred to produce 
a second generation of flies which had developed in $1.0 \mathrm{mg} \mathrm{mL} \mathrm{m}^{-1}$ tetracycline-treated media. Fecund females obtained after both one and two generations of tetracycline exposure were tested with the PCR assay to determine if there were detectable levels of Wolbachia. Progeny derived from both levels of tetracycline exposure were then bred on standard, tetracycline-free maize medium for three generations (20 males and 20 females in each generation) in order to reduce the possibility of decreased fecundity resulting from maternal exposure to tetracycline.

\section{Test for fertility effects}

After three generations of maintenance on tetracycline-free medium, uninfected individuals from both levels of tetracycline exposure were used in reciprocal crosses with infected individuals. Crosses were conducted by pairing single males with single females. Mating and oviposition on the medium was allowed to occur for $120 \mathrm{~h}$. For each of the four possible crosses between infected and uninfected individuals, 20 replicates were established for flies derived from one generation of tetracycline exposure, and 15 replicates for flies derived from two generations of exposure.

An effect of Wolbachia on fertility would be indicated by an association between the presence or absence of pupae and infection. Therefore, a multiway contingency table analysis was conducted on the number of fertile and infertile pairs from each treatment using SYSTAT v.5.2 (Wilkinson, 1989). Fertility (pupae or no pupae) was used as the response variable and the level of antibiotic exposure (one or two generations), female condition (infected or uninfected), and male condition (infected or uninfected) were used as explanatory variables in a four-dimensional analysis. The significance of each potential interaction was determined by testing the difference $\chi^{2}$, calculated by finding the difference between likelihood-ratio $\chi^{2}$ statistics of two models differing in only one term (Fienberg, 1981).

Reproductive performance between crosses may be influenced by several factors other than Wolbachia. First, tetracycline-treated media yielded relatively few offspring. Therefore, inbreeding may have occurred for four generations prior to the reciprocal crosses and could be responsible for fertility differences between infected and uninfected individuals. Secondly, the two treated lines may differ genetically because of stochastic effects of small population size following tetracycline exposure. Thirdly, antibiotic toxicity has an effect on the survival of eggs and could potentially influence the reproductive biology of adults.

We explored the potential effects of these factors by allowing Wolbachia populations to recover (confirmed by PCR) in a subset of treated individuals which were then used in reciprocal crosses with individuals that were untreated and known to be infected. A multiway contingency table analysis was then used to determine if there was an association between fertility, presence of Wolbachia and infection status. If reproductive effects are the result of any of these factors, then reproductive performance should not change in this second set of crosses. If, however, the effects are associated with Wolbachia, then the two sets of crosses should exhibit a difference in fertility.

\section{Test for fecundity effects}

In fertile crosses between infected and uninfected individuals, an effect of Wolbachia on egg production or development would be indicated by inequalities in mean daily pupal production between reciprocal crosses. Analysis of variance was used to determine if heterogeneity existed between crosses in pupal production. Fisher's Paired Least Significant Difference test (PLSD) was used to identify significant differences between crosses in pupal production.

\section{Results}

\section{Screening for Wolbachia}

The results of the PCR screen for the Wolbachia fts $Z$ gene are presented in Table 1. Four positive amplifications were detected. Three species from the genus Sphyracephala and Eurydiopsis subnotata were found to be positive for Type A Wolbachia, but negative for Type B Wolbachia. Amplification products of the appropriate length, 1035-1047 bases, were present for all four species. Neither Type A nor Type B Wolbachia was detected in $C$. dalmanni or $C$. whitei; thus, the microbes do not contribute to the observed female-biased sex ratio distortion in these two species.

\section{Fertility effects}

Comparison of the fertility of flies from reciprocal crosses between infected and uninfected strains (Table 2) revealed a significant association between fertility and the presence of infection in males (diff. $\left.\chi_{1}^{2}=11.53, P<0.005\right)$, with $43 \%$ of 70 uninfected males failing to produce pupae compared to only

(C) The Genetical Society of Great Britain, Heredity, 81, 254-260. 
Table 1 PCR amplification of Wolbachia ftsZ gene in 17 species of stalk-eyed flies

\begin{tabular}{lll}
\hline Genus & Species & Wolbachia* \\
\hline Cyrtodiopsis & dalmanni & Absent \\
& whitei & Absent \\
& quinqueguttata & Absent \\
Teleopsis & breviscopium & Absent \\
& quadriguttata & Absent \\
& rubicunda & Absent \\
Eurydiopsis & subnotata & Present \\
Diasemopsis & aethiopica & Absent \\
& dubia & Absent \\
& munroi & Absent \\
& sylvatica & Absent \\
Diopsis & apicalis & Absent \\
& fumipennis & Absent \\
Sphyracephala & detrahens & Absent \\
& beccarii & Present \\
& brevicornis & Present \\
& munroi & Present \\
\hline
\end{tabular}

*Positive amplifications are Type A.
$17 \%$ of 70 infected males. No significant association was detected between fertility and presence of the infection in females (diff. $\chi_{1}^{2}=0.55, P>0.10$ ) or fertility and number of generations of tetracycline exposure (diff. $\chi_{1}^{2}=3.55, P>0.05$ ). Consequently, results from the two levels of tetracycline exposure were combined in subsequent analyses of fertility.

After recovery of Wolbachia in a subset of antibiotic-treated individuals, the number of infertile males decreased from $43 \%$ to $18 \%$ (Table 3 ). A multiway contingency table analysis revealed a significant association between fertility and the presence of Wolbachia in males (diff. $\chi_{1}^{2}=6.66, P<0.01$ ), but not in females (diff. $\chi_{1}^{2}=4.09, P>0.10$ ). This analysis also indicated that the fertility effect observed in the first set of crosses was not present in the second set where there was recovery of Wolbachia (diff. $\left.\chi_{1}^{2}=2.03, P>0.10\right)$.

\section{Fecundity effects}

Analysis of variance failed to reveal any significant differences $\left(F_{3,87}=1.7, P=0.17\right)$ in pupal production between fertile reciprocal crosses (Table 4). However, there was a significant difference

Table 2 Fertility of reciprocal crosses between strains of Sphyracephala beccarii infected and uninfected with Wolbachia

\begin{tabular}{llrrrr}
\hline & & \multicolumn{3}{c}{ Cross type (female $\times$ male) } \\
\cline { 3 - 6 } $\begin{array}{l}\text { Tetracycline } \\
\text { exposure }\end{array}$ & Fertility & $\mathrm{I} \times \mathrm{U}$ & $\mathrm{U} \times \mathrm{I}$ & $\mathrm{I} \times \mathrm{I}$ & $\mathrm{U} \times \mathrm{U}$ \\
\hline One generation & Pupae present & 9 & 16 & 16 & 10 \\
& Pupae absent & 11 & 4 & 4 & 10 \\
\multirow{2}{*}{ Two generations } & Pupae present & 11 & 11 & 15 & 10 \\
& Pupae absent & 4 & 4 & 0 & 5 \\
\hline
\end{tabular}

I, infected; U, uninfected.

Table 3 Comparison of the fertility of reciprocal crosses between strains of Sphyracephala beccarii infected and uninfected with Wolbachia. Wolbachia $(+)$ strains treated with tetracycline had subsequent recovery of Wolbachia

\begin{tabular}{llrrrr}
\hline $\begin{array}{l}\text { Infection status } \\
\text { of treated } \\
\text { individuals }\end{array}$ & & \multicolumn{4}{c}{ Cross type (female $\times$ male) } \\
\cline { 3 - 6 } & Fertility & $\mathrm{I} \times \mathrm{T}$ & $\mathrm{T} \times \mathrm{I}$ & $\mathrm{I} \times \mathrm{I}$ & $\mathrm{T} \times \mathrm{T}$ \\
\hline Wolbachia $(-)$ & Pupae present & 20 & 27 & 31 & 20 \\
& Pupae absent & 15 & 8 & 4 & 15 \\
Wolbachia (+) & Pupae present & 15 & 17 & 13 & 18 \\
& Pupae absent & 5 & 3 & 7 & 2 \\
\hline
\end{tabular}

Wolbachia $(-)$, absent; $(+)$, present. I, infected (not treated with tetracycline); $\mathrm{T}$, treated with tetracycline. Values are totals from Table 2. 
Table 4 Mean daily pupal production $( \pm \mathrm{SE})$ per pair for all fertile reciprocal crosses between strains of Sphyracephala beccarii infected and uninfected with Wolbachia

\begin{tabular}{lcccc}
\hline \multirow{2}{*}{$\begin{array}{l}\text { Tetracycline } \\
\text { exposure }\end{array}$} & \multicolumn{4}{c}{ Cross type $($ female $\times$ male) } \\
\cline { 2 - 5 } & $\mathrm{I} \times \mathrm{U}$ & $\mathrm{U} \times \mathrm{I}$ & $\mathrm{I} \times \mathrm{I}$ & $\mathrm{U} \times \mathrm{U}$ \\
\hline One generation & $\begin{array}{c}10.8 \pm 5.0 \\
(n=9)\end{array}$ & $\begin{array}{l}8.3 \pm 1.8 \\
(n=15)\end{array}$ & $\begin{array}{c}14.9 \pm 2.2 \\
(n=16)\end{array}$ & $\begin{array}{c}10.7 \pm 1.9 \\
(n=10)\end{array}$ \\
Two generations & $\begin{array}{l}8.4 \pm 0.6 \\
(n=11)\end{array}$ & $\begin{array}{c}8.0 \pm 0.9 \\
(n=12)\end{array}$ & $\begin{array}{c}9.0 \pm 1.0 \\
(n=15)\end{array}$ & $\begin{array}{c}6.3 \pm 1.1 \\
(n=10)\end{array}$ \\
\hline
\end{tabular}

I, infected; U, uninfected.

$\left(F_{1,89}=5.0, \quad P=0.03\right)$ in mean pupal production between lines receiving different levels of tetracycline exposure. Mean daily pupal production per pair was $11.3 \pm 1.3(\mathrm{SE})$ in lines with one generation of tetracycline exposure and $8.0 \pm 0.5$ in lines with two generations of exposure. There was no interaction between cross type and level of tetracycline exposure for pupal production $\left(F_{1,3}=0.8, P=0.47\right)$. PCR analysis of uninfected females used in all crosses confirmed that these treated strains were free of Wolbachia.

\section{Discussion}

The pattern of Wolbachia infection in the 17 species of stalk-eyed flies screened, with three of the four positive amplifications occurring in the genus Sphyracephala, suggests the existence of a unique relationship between the microbe and these species. Assuming the overall probability of infection in diopsids is $23 \%(4 / 17)$, the probability that at least three of four Sphyracephala harbour the infection by chance is only 0.04 . There are two possible scenarios which can explain this nonrandom pattern of infection. First, the Wolbachia may have been acquired prior to the divergence of the Sphyracephala genus and then subsequently lost in S. detrahens. Secondly, given the high rate of horizontal transmission of Type A Wolbachia, the flies in this genus may have a predisposition for horizontal acquisition of the microbe and have been infected independently of each other. Although flies in the genus Sphyracephala can be found on several continents, all of the infected species are commonly found in dense aggregations near streams (Feijen, 1989). Possible sources of infection include predatory mites (Johanowicz \& Hoy, 1995) and parasitoid wasps (Feijen \& Schulten, 1981a,b). Determination of Wolbachia fts $Z$ sequence divergence in these species is needed to differentiate between these two potential routes of infection.

Our results did not reveal any differences in pupal production between fertile crosses. Specifically, fertile crosses between uninfected females and infected males, which have significantly lower fecundity in systems exhibiting CI, did not produce significantly different levels of pupae from all other crosses. Thus, we can conclude that Wolbachia in this species do not cause CI or that the level of CI is sufficiently weak not to be detected by this assay. The absence of CI in this system is not surprising. For example, although some Wolbachia-infected populations of Drosophila simulans exhibit CI, others do not (Hoffmann et al., 1996).

The observed reduction in fecundity in lines receiving two generations of tetracycline exposure in comparison to those receiving only one, is most likely an artifact of the crosses being conducted at two different times. Slight environmental differences, such as media quality or laboratory temperature, could translate into significant effects on both frequency of mating and survival of offspring.

Interestingly, the results of our crosses are consistent with an association between the microbes and enhanced male fertility. Approximately $43 \%$ of uninfected males exhibit infertility compared to only $17 \%$ of infected males. This pattern is also evident in treated flies that did not lose Wolbachia compared to those that did. Positive effects of Wolbachia on host productivity have been reported for Trichogramma bourarachae (Girin \& Bouletreau, 1995), Tribolium confusum (Wade \& Chang, 1995), Nasonia vitripennis (Stolk \& Stouthamer, 1996) and D. simulans (Poinsot \& Mercot, 1997).

The male fertility effect we found in $S$. beccarii is unlikely to be caused by inbreeding depression, line divergence or antibiotic toxicity. A multiway contin- 
gency analysis revealed a significant association between fertility and the presence of Wolbachia in males, suggesting that the bacteria, rather than these other factors, are responsible for the observed fertility effect. Further evidence against inbreeding depression as the cause of the $26 \%$ reduction in male fertility comes from a study of artificial selection in another stalk-eyed fly, Cyrtodiopsis dalmanni (Wilkinson et al., 1998). After 22 generations of selection with an effective population size of 29 (coefficient of inbreeding estimated to be 53\%), male fertility declined $10 \%$ in comparison to an outbred stock population (Wilkinson, unpubl. data). In contrast, a comparable estimate of the inbreeding coefficient for the $S$. beccarii used in the reciprocal crosses reported here was 5\%. Nevertheless, it remains possible that other genetic changes in the flies could have caused a paternal fertility effect. Introgression of the uninfected genotype into the infected background is needed to address this possibility further.

One possible explanation for the male fertility effect in $S$. beccarii is that the bacteria were originally CI producers which spread to near fixation, after which the host genome accommodated to their presence by adjusting spermatogenesis to reduce the negative effects of CI in males. By eliminating the microbes with antibiotics, spermatogenesis may have been altered causing reduced fertility. Turelli (1994) has suggested that the host genome can be selected to suppress microbial effects in males, whereas suppression in females is generally not favoured. In addition, recent molecular studies have illustrated that Wolbachia interact with centrosomal microtubules and host proteins, both important in gametogenesis (Kose \& Karr, 1995). These interactions may provide a potential mechanism by which Wolbachia alter the reproductive biology of their hosts. Studies designed to determine how the bacteria influence spermatogenesis are needed to confirm this hypothesis.

\section{Acknow ledgements}

We would like to thank Marion Kotrba, Daven Presgraves and Paul Reillo for collection of flies; Sharvari Bhatt and Lili Crymes for maintenance of laboratory populations and Renee Goodwin for PCR assays; Daven Presgraves and two anonymous reviewers for helpful comments on earlier versions of this manuscript. This research was supported by grants from the National Science Foundation to J.H.W. and G.S.W.

\section{References}

BURKHARDT, D. AND MOTTE, I. DE LA 1983. How stalk-eyed flies eye stalk-eyed flies: observations and measurements of the eyes of Cyrtodiopsis whitei (Diptera, Diopsidae). J. Comp. Physiol. A, 151, 407-421.

FEIJEN, H. 1989. Diopsidae. In: Griffiths, G. C. D. (ed.) Flies of the Nearctic Region, pp. 1-122. E. Schweizerbart'sche Verlagsbuchhandlung, Stuttgart.

FEIJEN, H. AND SCHULTEN, G. G. M. 1981a. Egg parasitoids (Hymenoptera: Trichogrammatidae) of Diopsis macrophthalma (Diptera: Diopsidae). Neth. J. Zool., 31, 381-347.

FEIJEN, H. AND SCHUlten, G. G. M. 1981b. Egg parasitoids of rice pests in Malawi, East Africa. Int. Rice Res. Newsl., 6, 17-18.

Fienberg, s. E. 1981. The Analysis of Cross-Classified Categorical Data. MIT Press, Cambridge, MA.

FRANK, S. A. 1996. Host-symbiont conflict over the mixing of symbiotic lineages. Proc. R. Soc. B, 263, 339-344.

GIRIN, C. AND BOUletreAU, M. 1995. Microorganismassociated variation in host infestation efficiency in a parasitoid wasp, Trichogramma bourarachae (Hymenoptera: Trichogrammatidae). Experientia, 51, 398-401.

HOFFMANN, A. A. AND TURELl, M. 1988. Unidirectional incompatibility in Drosophila simulans: inheritance, geographic variation and fitness effects. Genetics, 119, 435-444.

HOFFMANN, A. A., ClANCY, D. J. AND DUNCAN, J. 1996. Naturally-occurring Wolbachia infection in Drosophila simulans that does not cause cytoplasmic incompatibility. Heredity, 76, 1-8.

HOFFMANN, A. A., TURELli, M. AND hARShMAN, L. 1990. Factors affecting the distribution of cytoplasmic incompatibility in Drosophila simulans. Genetics, 126, 933-948.

JOHANOWICZ, D. L. AND HOY, M. A. 1995. Molecular evidence for A-Wolbachia endosymbiont in the predatory mite Metaseiulus occidentalis. J. Cell. Biochem., 21, 198.

KOSE, H. AND KARR, T. L. 1995. Organization of Wolbachia pipientis in the Drosophila fertilized egg and embryo revealed by an anti-Wolbachia monoclonal antibody. Mech. Dev., 51, 275-288.

LORCH, P., WILKINSON, G. AND REILlo, P. 1993. Copulation duration and sperm precedence in the stalk-eyed fly Cyrtodiopsis whitei (Diptera: Diopsidae). Behav. Ecol.. Sociobiol., 32, 303-311.

O'NEILL, S. L. 1995. Wolbachia pipientis: symbiont or parasite? Parasitol. Today, 11, 168.

POINSOT, D. AND MERCOT, H. 1997. Wolbachia infection in Drosophila simulans: does the female host bear a physiological cost? Evolution, 51, 180-186.

PRESGRAVES, D. C., SEVERANCE, E. AND WILKINSON, G. S. 1997. Sex chromosome meiotic drive in stalk-eyed flies. Genetics, 147, 1169-1180.

Rousset, F., Bouchon, D., PINTUReau, B., JUCHAUlt, P. AND SOLIGNAC, M. 1992. Wolbachia endosymbionts responsible for various alterations of sexuality in arthro- 
pods. Proc. R. Soc. B, 250, 91-98.

STEYSKAL, G. 1972. A catalogue of species and key to genera of the family Diopsidae. Stuttg. Beitr. Naturkd., Ser. $A, 1-20$.

STOLK, C. AND STOUTHAMER, R. 1996. Influence of a cytoplasmic incompatibility-inducing Wolbachia on the fitness of the parasitoid wasp Nasonia vitripennis. Proc. Exp. Appl. Ent., 7, 33-37.

STOUTHAMER, R., BREEUWER, J., LUCK, R. AND WERREN, J. 1993. Molecular identification of microorganisms associated with parthenogenesis. Nature, 361, 66-68.

TURELLI, M. 1994. Evolution of incompatibility-inducing microbes and their hosts. Evolution, 48, 1500-1513.

WADE, M. AND CHANG, N. 1995. Increased male fertility in Tribolium confusum beetles after infection with the intracellular parasite Wolbachia. Nature, 373, 72-74.

Werren, J. 1997. Biology of Wolbachia. Ann. Rev. Ent., 42, 587-609.
WERREN, J. AND JAENIKE, J. 1995. Wolbachia cytoplasmic incompatibility in mycophagous Drosophila and their relatives. Heredity, 74, 320-326.

WERREN, J., GUO, L. AND WINDSOR, D. W. 1995b. Distribution of Wolbachia in neotropical arthropods. Proc. $R$. Soc. B, 262, 147-204.

WERREN, J., ZHANG, W. AND GUO, L. 1995a. Evolution and phylogeny of Wolbachia: reproductive parasites of arthropods. Proc. R. Soc. B, 261, 55-71.

WILKINSON, G. s. 1993. Artificial selection alters allometry in the stalk-eyed fly Cyrtodiopsis whitei (Diptera: Diopsidae). Genet. Res., 62, 213-222.

WILKINSON, G. S., PRESGRAVES, D. C. AND CRYMES, L. 1998. Male eye span in stalk-eyed flies indicates genetic quality by meiotic drive suppression. Nature, 391, 276-279.

WILKINSON, L. 1989. SYSTAT: the system for statistics. Systat, Evanston, IL. 